Original Article (short paper)

\title{
Verbal instruction does not change myoelectric activity during seated row exercise in trained and untrained men
}

\author{
Rafael A. Fujita ${ }^{1}$, Pedro U. De Marchi ${ }^{1}$, Nilson R. S. Silva ${ }^{1}$, Matheus M. Gomes ${ }^{1}$ (iD \\ ${ }^{1}$ Universidade de São Paulo, Escola de Educação Física e Esporte de Ribeirão Preto, \\ Laboratório de Biomecânica e Controle Motor, Ribeirão Preto, SP, Brasil
}

\begin{abstract}
Aims: This study aimed to analyze the effect of verbal instruction on myoelectric activity (EMG) of the elbow flexors and shoulder extensors muscles during seated row exercise at 70\%1RM, in trained and untrained individuals. Methods: The study sample comprised of seven males ( $21 \pm 1.9$ years) who had not practiced resistance training for at least one year (untrained group), and eight males ( $23 \pm 2.6$ years) who had regularly practice strength training for $2.4 \pm 0.7$ years (trained group). All individuals performed the seated row exercise with $70 \% 1 \mathrm{RM}$ in conditions with and without verbal instruction. The EMG of the latissimus dorsi, teres major, posterior deltoid, triceps brachii long head and biceps brachii were measured. Results: There was no effect of verbal instruction on EMG at any muscle analyzed, for both groups. There was a difference between groups only for the teres major and triceps brachii $(\mathrm{p}<0.05)$. Untrained individuals had greater myoelectric activity in teres major and triceps brachii long head muscles compared to the trained group during the exercise.
\end{abstract}

Keywords: electromyography; kinesiology; attentional focus; muscle recruitment; muscle activation.

\section{Introduction}

Regarding strength training, there is an interest in muscle recruitment during the exercises ${ }^{1-3}$. On this kind of training, the verbal instruction (VI), composed by small phrases, helps to direct the practitioner to contract a specific muscle or muscle group ${ }^{4}$, as an attempt to emphasize its participation (e.g., focus on the contract the back muscles instead of the arms muscles). Recent studies have highlighted the importance of VI as a way to increase the myoelectric activity (EMG) $)^{1,4,5}$. Snyder and $\mathrm{Leech}^{4}$ showed that VI focusing on the back musculature during the lat pulldown exercise, made the participants increase the latissimus dorsi activity in $17.6 \%$. Corroborating these results, Calatayud, Vinstrup, Jakobsen, Sundstrup, Colado, Andersen ${ }^{6}$ showed that VI focused on the pectoralis major during pushing exercise increased its muscle activity by $9 \%$. However, the literature indicates that the effects of VI on muscle activity depend upon the intensity of the exercise ${ }^{7}$.

Previous studies revealed that VI increased EMG in the target muscles when the intensity was lower than $60 \% 1 \mathrm{RM}^{1,4,5}$ however, at $80 \% 1 \mathrm{RM}$, the effect of VI was smaller ${ }^{1}$ or did not exist $^{2}$. Snyder and Fry ${ }^{1}$ have shown that soccer athletes were able to increase muscle activity with VI during the bench press at $50 \% 1 \mathrm{RM}$. When focusing on the pectoralis major muscle, the participants had a $22.3 \%$ increase in EMG for the same muscle, as well when it was for the triceps brachii, participants showed a $25.7 \%$ increase on EMG. When the intensity was set at $80 \%$ 1RM, the VI showed less effect to emphasize the target muscles, creating an increase on EMG only on the shoulder adductors (pectoralis major and anterior deltoid).

Seeking to better understand the relationship between load intensity and the effects of VI on EMG, Calatayud et al. ${ }^{5}$ analyzed the myoelectric activity of the pectoralis major and triceps brachii while executing the bench press at intensities of 20,30, 40, 50, 60 and $80 \% 1 \mathrm{RM}$. While hearing VI to emphasize the pectoralis major or to emphasize the triceps brachii, the participants increased their EMG of both muscles at intensities between 20 and $60 \% 1 \mathrm{RM}$. Although there were no increases at $80 \% 1 \mathrm{RM}$, corroborating previous study ${ }^{2}$.

It is evident that there is a gap in the effect of VI on the EMG while performing exercises at 70\% 1RM. Therefore, investigating the contribution of VI at this intensity is crucial once this relative intensity is commonly used in strength training programs $^{8}$. Also, there is no evidence related to the effect of VI at $70 \% 1 \mathrm{RM}$ on individuals with different training levels. Analyze if a different level of experience on strength training is capable of modifying the EMG through VI become relevant, as the results could reflect on how physical education and physiotherapy professionals guide their clients (with and without experience on strength training).

Thus, the present study sought to analyze the effect of VI on EMG of the elbow flexors and shoulder extensors muscles during the seated row exercise, on trained and untrained individuals. It was hypothesized that both groups would increase EMG activity in the latissimus dorsi and teres major muscles while re- 
ceiving VI. Furthermore, it was hypothesized that the trained group would show greater improvements.

\section{Methods}

\section{Experimental approach to the problem}

This study aimed to answer two questions that were not clarified yet: (a) What is the effect of verbal instruction to modify the myoelectric activity during exercise at a load of $70 \%$ 1RM? (b) How can verbal instruction influence myoelectric activity in individuals with different levels of strength training?

To answer this questions, electromyographic data of the muscles latissimus dorsi (LD), teres major (TM), posterior deltoid (PD), triceps brachii long head (TB) and biceps brachii (BB) of dominant limb were collected during the seated row exercise while performing maximum repetitions in two different conditions: without verbal instruction (NONE) and with verbal instruction (V-I). The verbal instruction given was to emphasize the shoulder extension as an attempt to elevate the myoelectric activity of LD and TM muscles.

\section{Subjects}

The study sample was composed of 15 males. Participants were divided into 2 groups: untrained $(n=7)$ and trained $(n=8)$. The untrained group had a maximum of 6 months strength training experience and were not training in the last 12 months. The trained group had been strength training for a minimum of 12 months (Table 1).

None of the participants presented historic of orthopedic lesions that could impair the performance of the tests, as well as neurological problems or any other health problem that would interfere with the accomplishment of the tasks proposed in the present study. They were oriented about the experimental protocols and signed the consent term to participate in this study, which is following the 1964 Helsinki Declaration. This study was approved by the local Ethics Committee (process number 1.940.743 - CAAE: 63679516.7.0000.5659).

Table 1 - Mean \pm standard deviation of age, body mass, body height, and experience with the resistance training of the participants.

\begin{tabular}{lcc}
\hline & $\begin{array}{c}\text { Participants } \\
\text { Untrained } \\
\text { Group }(\mathbf{n}=7)\end{array}$ & $\begin{array}{c}\text { Trained } \\
\text { Group (n= 8) }\end{array}$ \\
\hline Age (years) & $21.0 \pm 1.9$ & $23.0 \pm 2.6$ \\
Body Mass (kg) & $73.3 \pm 11.4$ & $79.8 \pm 7.2$ \\
Body Height (cm) & $177.9 \pm 4$ & $179.6 \pm 4.5$ \\
$\begin{array}{l}\text { Experience with resistance } \\
\text { training (years) }\end{array}$ & $0.2 \pm 0.1$ & $2.4 \pm 0.7$ \\
$\begin{array}{l}\text { Weight lifted in seated row } \\
\text { exercise (kg) }\end{array}$ & $43.0 \pm 4.3$ & $58.5 \pm 16.3$ \\
$\begin{array}{l}\text { Number of repetitions in } \\
\text { seated row exercise }\end{array}$ & $10.9 \pm 1.2$ & $11.1 \pm 1.2$ \\
\hline
\end{tabular}

\section{Procedures}

Before data collection, the reproducibility of main measuring instruments was evaluated in five subjects into two separate days. The intraclass correlation coefficients (ICCs) values showed that the technical error of measurement was 1,04\%. Data collection was done on three different days (load quantification test, Test 1, Test 2) at the Kinanthropometry and Human Development Laboratory of the local institution. Initially, the interval between each test was stipulated to two to seven days ${ }^{9}$. The interval between each test was about $4.45 \pm 2.25$ days.

Before the load quantification test, the participants received explanations about the procedures and signed the consent term. Once there were no references for the resistance (weight) tolerated by the participants, we estimated the $1 \mathrm{RM}$ as $75 \%$ of the participant body weight following previous recommendations ${ }^{10}$. For both warm-up and load quantification, the movement cadence was set to two seconds for concentric and two seconds for eccentric movement ${ }^{1,5}$, controlled by a metronome app (Pro metronome, Xanin Technology, China). Therefore, the participants went through a warm-up on the seated-row machine (Flex Fitness Equipment, SP, Brazil) composed of 2 sets of 12 repetitions with 40\% 1RM. After the warm-up, a five minutes interval was given, then the participants went through individual load quantification. The load quantification was done by accounting the maximal repetitions until failure at $70 \% 1 \mathrm{RM}$, respecting the proposed cadency. We utilized the Brzycki ${ }^{11}$ equation to predict 1RM percentages.

After the load quantification test, Test 1 investigated the EMG during the seated-row in NONE condition. In this condition, none VI was provided to the participants. During the seated-row, the participants remained seated, with the chest against the equipment support. At the initial position, the elbows were extended, and the shoulders flexed, then on the concentric phase of the exercise the shoulders extended, and the elbows flexed. During the movement, the forearm was always in a supinated position. In this position, the biceps brachii has a better performance in the elbow flexion ${ }^{12}$.

In Test 1 , before starting any task, the same warm-up used in the load quantification test was performed at $40 \% 1 \mathrm{RM}^{13}$. After warming-up and five minutes interval ${ }^{14}$, the maximum voluntary isometric contraction (MVIC) was collected. For each movement, 3 sets of 8 seconds in maximal contraction with 90 seconds intervals were performed. The MVIC was done for the latissimus dorsi and teres major muscles ${ }^{15}$, posterior deltoid muscle $^{14}$, triceps brachii long head, and biceps brachii muscles $^{16,17}$ as indicated by the literature. In all MVIC, the participants were verbally motivated to produce as much force as they could $^{18,19}$. At the end of all MVIC, there were five minutes of passive interval. Then the participants performed 2 sets of 8 to 12 repetitions at the seated-row machine, seeking concentric failure at $70 \% 1 \mathrm{RM}$ with a five minutes passive interval between each set.

In Test 2, the EMG was evaluated in the seated row exercise in the V-I condition. The protocol was the same as in Test 1 , with the addition of the VI "Concentrate on extending the shoulders, pull with the back." This VI was provided at the beginning 
of the repetitions and every three repetitions during the two sets of movements. The instruction was short and simple similarly to those used in previous studies ${ }^{1,2,4,5}$. In addition to the VI, the participant received palpation at the region of the latissimus dorsi muscle on the back, following the recommendations of Snyder and Leech ${ }^{4}$.

In both Tests 1 and 2 (NONE and V-I conditions), all repetition was counted using the same cadence used on the load quantification test. The number of repetitions performed in all sets was similar between the groups (Table 1). Among the conditions, the mean number of repetitions was also similar (11.08 \pm 0.98 to NONE, and $10.87 \pm 1.33$ for $\mathrm{V}-\mathrm{I}$ ).

For EMG data collection, wireless electromyography (Trigno Wireless, Delsys, Natick, MA, USA) composed by 16 channels of 16 bits resolution was used. The skin hairs were scraped, and the skin was rubbed with a disposable wipe with $70 \%$ anhydrous ethyl alcohol, aiming to minimize the skin's impedance before attaching any surface electrode. The surface electrodes then were fixed on the skin, positioned over the latissimus dorsi, teres major, posterior deltoid, triceps brachii long head and biceps brachii of the dominant limb. We chose to analyze these muscles because they are the main agonists of the shoulder extension and elbow flexion movements that occur in the seated-row exercise. Although the brachialis muscle is an important elbow flexor, it was not possible to analyze it because of noise in the electromyographic signal of this deep muscle. For the triceps brachii long head and biceps brachii muscles, the electrodes were positioned following SENIAM recommendations $^{20}$. The fixation of the surface electrodes over the remaining muscles followed recommendations from the previous articles $^{14,21,22}$. Data were collected at a $2000 \mathrm{~Hz}$ frequency.

\section{Electromyographic data processing}

The EMG data were filtered using the Butterworth digital bandpass filter $(20 \mathrm{~Hz}-500 \mathrm{~Hz})$. To measure EMG, the root mean square (RMS) of each set was calculated (all repetitions together). The final RMS value represents the average of all repetitions realized in the two sets performed. Subsequently, these RMS values were normalized by the RMS value obtained during the MVIC of each muscle.

\section{Statistical analysis}

The data presented homogeneity of variance and homoscedasticity. Five analyses of variance (ANOVAs) were performed, having as factors: group (trained vs. untrained) and VI (with vs. without VI), the latter being treated as repeated measures. The dependent variables were the magnitudes (RMS) of EMG of the analyzed muscles. When necessary, Bonferroni post hoc tests were performed. The effect size (partial Eta square) and test power parameters were calculated. All statistical analyses were performed using the SPSS program (SPSS v.20, IBM Inc, Boston - MA, USA), maintaining the significance level at $\mathrm{p}<$ 0.05 .

\section{Results}

In general, the results did not show the effect of VI on the EMG of the analyzed muscles in both groups (trained and untrained individuals), but they pointed out differences between groups in some muscles.

For latissimus dorsi muscle, ANOVA did not indicate differences between groups $\left[\mathrm{F}_{1,12}=0.251, \mathrm{p}=0.626\right.$, effect size $=$ 0.020 , power $=0.075]$ nor for VI conditions $\left[F_{1,12}=0.148\right.$, $\mathrm{p}=0.707$, effect size $=0.012$, power $=0.065]$. Both groups showed similar activity in NONE and V-I conditions (trained $79.85 \pm 13.68 \%$, untrained 83.31 $\pm 18.85 \%$ ) (Figure 1).

For teres major muscle, ANOVA indicated difference for group $\left[\mathrm{F}_{1,10}=10.030, \mathrm{p}=0.010\right.$, effect size $=0.501$, power $=$ $0.814]$ but did not show effect for VI $\left[\mathrm{F}_{1,10}=0.010, \mathrm{p}=0.922\right.$, effect size $=0.001$, power $=0.051]$. The untrained participants

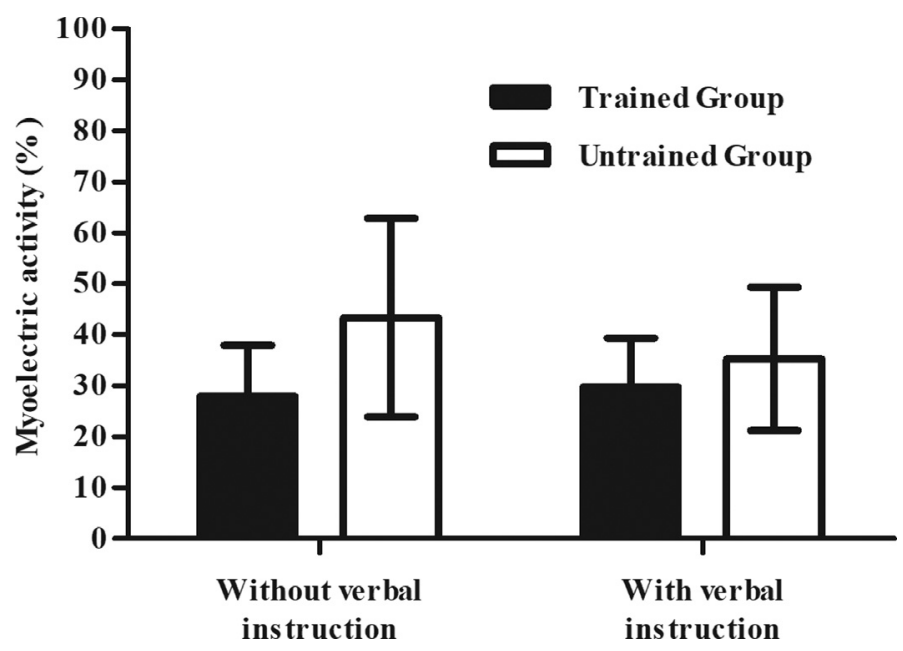

Figure 1 - Myoelectric activity of the latissimus dorsi muscle during the seated row at $70 \% 1 \mathrm{RM}$ with and without verbal instructions in trained and untrained group.

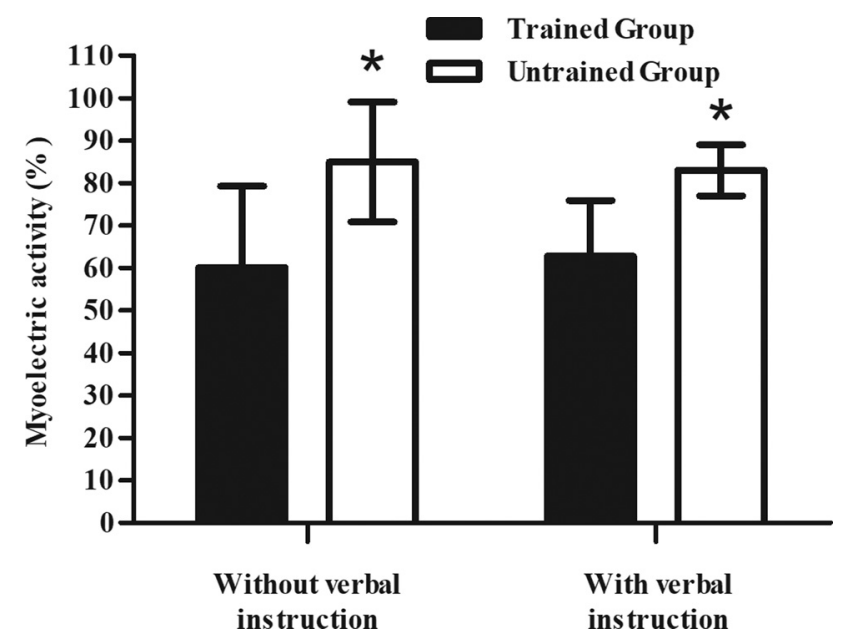

Figure 2 - Myoelectric activity of the teres major muscle during the seated row at $70 \% 1 \mathrm{RM}$ with and without verbal instructions in trained and untrained group. $(*$ indicates bigger than trained group, $\mathrm{p}<0.05)$. 
showed higher EMG on teres major muscle $(83.96 \pm 10.06 \%)$ than the trained participants $(61.43 \pm 16.13 \%)$ (Figure 2$)$.

Regarding triceps brachii long head muscle, ANOVA showed effect for group $\left[\mathrm{F}_{1,11}=13.241, \mathrm{p}=0.004\right.$, effect size $=$ 0.546 , power $=0.911]$, but did not indicate difference for VI $\left[\mathrm{F}_{1,11}=0.358, \mathrm{p}=0,562\right.$, effect size $=0.032$, power $\left.=0.085\right]$ and showed interaction between group and VI $\left[\mathrm{F}_{1,11}=5.715\right.$, $\mathrm{p}=0.036$, effect size $=0.342$, power $=0.587]$. Post-hoc tests showed that the untrained group presented higher EMG on the triceps brachii muscle $(31.95 \pm 8.80 \%)$ than the untrained group $(14.22 \pm 9.88 \%)$ in both conditions (Figure 3).

For biceps brachii muscle, ANOVA did not show difference for group $\left[\mathrm{F}_{1,10}=3.353, \mathrm{p}=0.089\right.$, effect size $=0.262$, power $=0.399]$ and VI $[\mathrm{F}(1,10)=1.290, \mathrm{p}=0.283$, effect size $=$ 0.114 , power $=0.177]$. The biceps brachii muscle showed similar activity in NONE and V-I conditions for both groups (trained $28.90 \pm 9.77 \%$, untrained $39.35 \pm 16.78 \%$ ) (Figure 4).

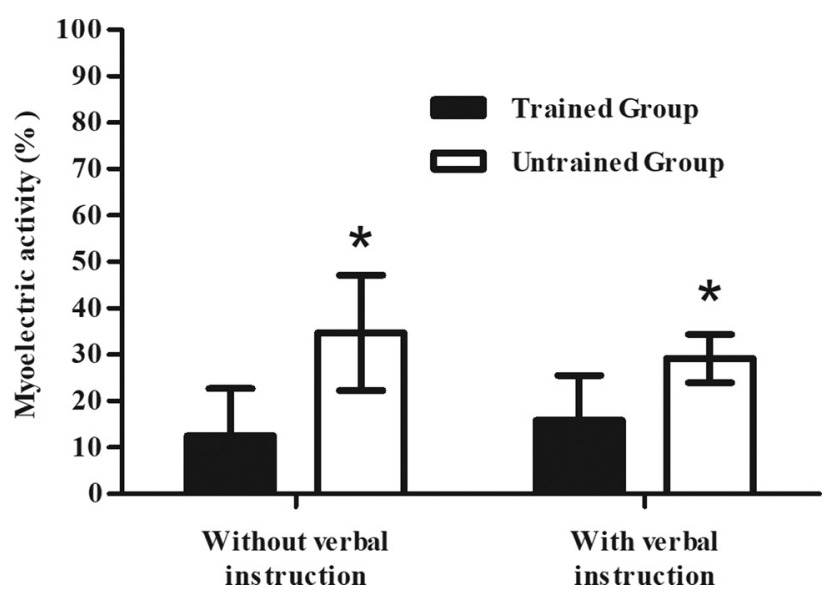

Figure 3 - Myoelectric activity of the triceps brachii long head muscle during the seated row at 70\% $1 \mathrm{RM}$ with and without verbal instructions in trained and untrained group. (* indicates bigger than trained group, $\mathrm{p}<0.05)$.

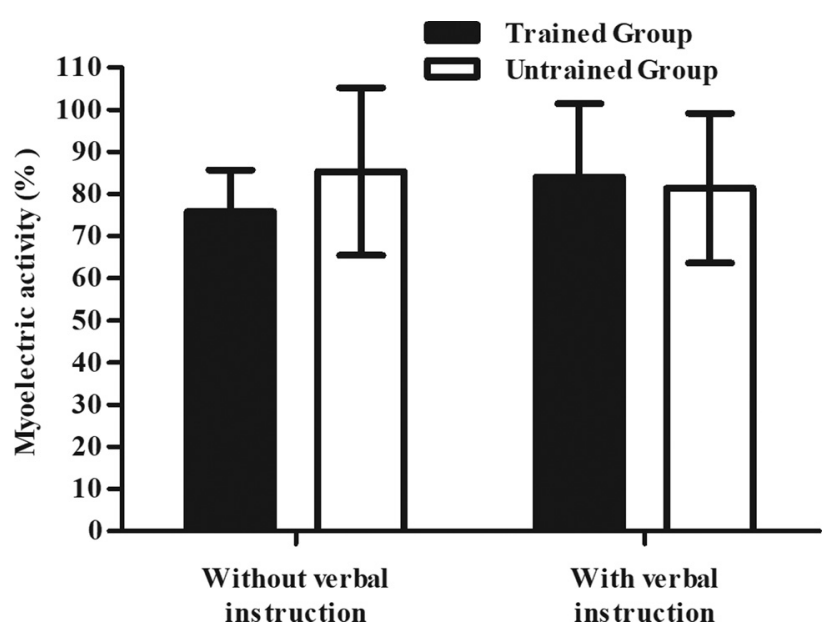

Figure 4 - Myoelectric activity of the biceps brachii muscle during the seated row at $70 \% 1 \mathrm{RM}$ with and without verbal instructions in trained and untrained group.

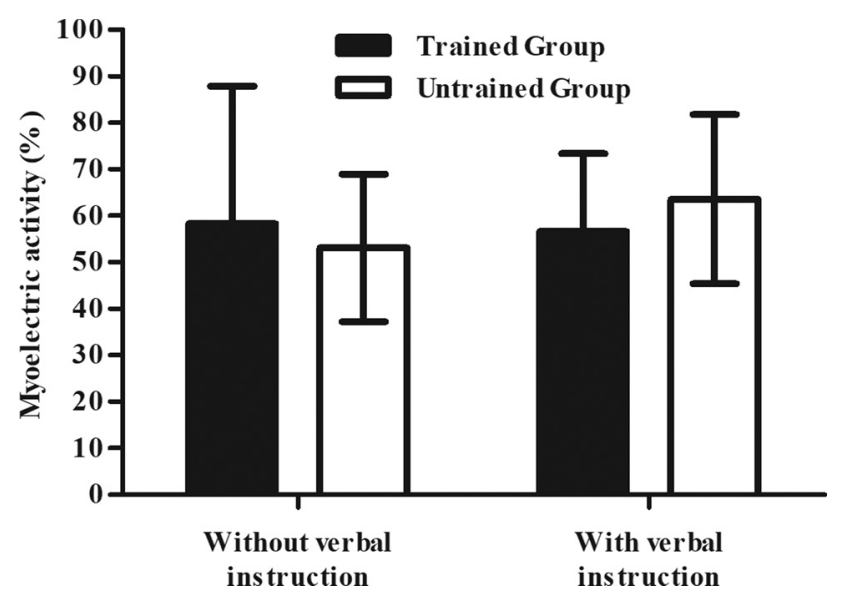

Figure 5 - Myoelectric activity of the posterior deltoid muscle during the seated row at $70 \% 1 \mathrm{RM}$ with and without verbal instructions in trained and untrained group.

Regarding to posterior deltoid muscle, ANOVA revealed no difference for group $\left[\mathrm{F}_{1,12}=0.009, \mathrm{p}=0.928\right.$, effect size $=$ 0.001 , power $=0.051]$ and VI $\left[\mathrm{F}_{1,12}=0.706, \mathrm{p}=0.417\right.$, effect size $=0.056$, power $=0.121]$. The posterior deltoid muscle showed similar EMG in NONE and V-I conditions for both groups (trained $57.43 \pm 23.20 \%$, untrained $58.34 \pm 17.02 \%$ ) (Figure 5).

\section{Discussion}

The present study analyzed the effect of VI on EMG of the elbow flexors and shoulder extensors muscles in trained and untrained individuals during the seated-row exercise at 70\% 1RM. There were doubts whether the efficiency of VI would exist at $70 \% 1 \mathrm{RM}$, a common intensity used in strength training programs $^{8}$. We hypothesized that both groups could increase the EMG while receiving the VI at this intensity. Also, we thought that the trained group would respond better to the VI than the untrained group. However, contrary to our hypothesis, the main result of the present study showed that VI did not increase EMG regardless of the training level.

Previous studies have shown that trained individuals increased their EMG of target muscles while receiving VI during resistance training exercises with submaximal intensities such as push-ups $^{6}$ and bench press ${ }^{23}$. Also, practitioners could increase the EMG of the target muscles while receiving VI in exercises with intensities up to $60 \% 1 \mathrm{RM}$ in untrained participants ${ }^{4}$ and trained participants ${ }^{1,5}$. Although the results found in these studies show the effectiveness of VI, we must take into account that the exercise intensities are considered as warm-up intensities ${ }^{13}$, which could explain the positive effect of VI. In higher intensities (e.g., $80 \% 1 \mathrm{RM}$ ), the VI had its effectiveness reduced ${ }^{1}$ or had no effect ${ }^{2,5}$ on increasing the EMG on target musculature.

Whilst the literature showed the influence of VI in different intensities of exercise; we found only one study that compared the effect of VI on different levels of training. Daniels and $\mathrm{Cook}^{2}$ analyzed the effect of VI in trained and untrained groups to emphasize the chest or arm muscles during the bench press ex- 
ercise realized with $80 \%$ 1RM. The results did not show significant interactions between EMG and groups. Trained and untrained men showed similar EMG of the agonist musculature with and without VI to focus on the pectoralis major or triceps brachii muscle.

Our results are following this study ${ }^{2}$. Even it was expected that VI would be able to increase the EMG of the latissimus dorsi and teres major muscles in both groups (trained and untrained), significant changes were not observed. The present results indicated that the VI "Concentrate on extending the shoulders, pull with the back" given to the participants did not increase LD and TM muscles EMG in both groups. Furthermore, once trained individuals have better intermuscular coordination ${ }^{24}$, we expected that EMG would change on the target muscles more effectively in the trained group. However, the results showed that both groups were not influenced by VI. Although, with a descriptive observation, the trained group increased EMG about $8.25 \%$ for the latissimus dorsi muscle and $2.73 \%$ for the teres major muscle with VI. On the other hand, the untrained group showed reductions of $3.92 \%$ for the latissimus dorsi muscle and $1.98 \%$ for the teres major muscle.

In contrast, the EMG of the triceps brachii long head and teres major muscles were different between the groups. The untrained group showed higher EMG for the teres major $(+22.53 \%)$ and triceps brachii long head $(+17.73 \%)$ when compared to the trained group. Possibly this was due to the low level of intermuscular coordination between two or more muscles, which causes higher recruitment of motor units, raising the EMG levels to the same motor task with the same relative inten$\operatorname{sity}^{25}$. Moreover, the untrained participants could be less used to this kind of effort and load intensity, resulting in a greater $\mathrm{EMG}^{2}$.

Indeed, we add information to the literature regarding the use of VI in the common intensity of $70 \% 1 \mathrm{RM}$, which is unable to increase EMG in both levels of training. Once previous studies $^{1,4,5}$ did not analyze the effect of VI in this intensity, our results clarify this lack in the literature and also reinforce that VI seems to be effective only in warm-up intensities like previous studies showed ${ }^{4,5}$. The absence of VI effect may be due to exercise intensity, once the exercise intensity is the key factor for changes in muscle activity ${ }^{7}$. Also, the increase in motor units recruitment is directly related to exercise intensity ${ }^{26}$. The higher demand for force production, the more motor units are recruited ${ }^{27}$. In this sense, the use of VI in exercise with high load did not increase the EMG of the target muscle ${ }^{5}$. According to Calatayud et al. ${ }^{5}$ in high exercise load, it is necessary for the recruitment of many motor units, then EMG increased occurs, and VI cannot further increase more such recruitment. Possibly at 70\% 1RM, this also occurred. Likely, the need to recruit more motor units for greater force production has made it difficult to selectively activate the muscles and activation of only the desired muscle/muscle group $^{4}$.

The present study has some limitations, such as the RMS interval analyzed. Our results showed the effect of VI on the average of RMS during all repetitions. Once the literature shows that the EMG increases as the end of the series approaches ${ }^{26,28}$, analyze the effect of VI in different intervals (e.g. initial repetitions versus final repetitions) among the series could be interest- ing to know which is the effect of VI in different intervals. Furthermore, in this article, the spectrum analyzes were not made, then future studies could investigate the firing rate of motor units to broaden understanding of the effect of verbal instruction on muscle recruitment.

\section{Conclusions}

The present study showed that the use of verbal instruction is not useful to increase EMG of target muscles in moderate intensities such as $70 \% 1 \mathrm{RM}$ regarding the level of training. In this sense, strength coaches and practitioners with different levels of strength training do not need to focus on promoting intentional activation to increase EMG of target muscles. Instead, coaches and practitioners could focus their attention on other aspects of resistance training such as posture, cadence, and movement coordination.

\section{References}

1. Snyder BJ, Fry WR. Effect of verbal instruction on muscle activity during the bench press exercise. J strength Cond Res. 2012;26(9):2394-2400. doi:10.1519/JSC.0b013e31823f8d11

2. Daniels RJ, Cook SB. Effect of instructions on EMG during the bench press in trained and untrained males. Hum Mov Sci. 2017;55:182-188. doi:10.1016/j.humov.2017.08.010

3. Vigotsky AD, Halperin I, Lehman GJ, Trajano GS, Vieira TM. Interpreting Signal Amplitudes in Surface Electromyography Studies in Sport and Rehabilitation Sciences. Front Physiol. 2017;8:985. doi:10.3389/fphys.2017.00985

4. Snyder BJ, Leech JR. Voluntary increase in latissimus dorsi muscle activity during the lat pull-down following expert instruction. J strength Cond Res. 2009;23(8):2204-2209. doi:10.1519/JSC.0b013e3181bb7213

5. Calatayud J, Vinstrup J, Jakobsen MD, Sundstrup E, Brandt M, Jay K, et al. Importance of mind-muscle connection during progressive resistance training. Eur J Appl Physiol. 2016;116(3):527-533. doi:10.1007/s00421-015-3305-7

6. Calatayud J, Vinstrup J, Jakobsen MD, Sundstrup E, Colado JC, Andersen LL. Mind-muscle connection training principle: influence of muscle strength and training experience during a pushing movement. Eur J Appl Physiol. 2017;117(7):1445-1452. doi:10.1007/s00421-017-3637-6

7. Stastny P, Golas A, Blazek D, Maszczyk A, Wilk M, Pietraszewski P, et al. A systematic review of surface electromyography analyses of the bench press movement task. PLoS One. 2017;12(2):e0171632. doi:10.1371/journal.pone.0171632

8. Fleck S, Kraemer W. Designing Resistance Training Programs. 4th ed. Campaing, IL: Human Kinetics; 2014.

9. Pirauá A, Barros Beltrão N, Ximenes Santos C, Pitangui A, Araújo R. Analysis of muscle activity during the bench press exercise performed with the pre-activation method on stable and unstable surfaces. Kinesiology. 2017;49. doi:10.26582/k.49.2.11

10. Guedes DP. Manual Prático Para Avaliação Em Educação Física. Editora Manole Ltda; 2006.

11. Brzycki M. Strength Testing-Predicting a One-Rep Max from Reps-to-Fatigue. J Phys Educ Recreat Danc. 1993;64(1):88-90. doi:10.1080/07303084.1993.10606684

12. Stewart OJ, Peat M, Yaworski GR. Influence of resistance, speed of movement, and forearm position on recruitment of the elbow flexors. Am J Phys Med. 1981;60(4):165-179. 
13. Kraemer W, Fry A. Strength Testing: Development and Evaluation of Methodology. Physiol Assess Hum Fit. January 1995:115-138.

14. Schoenfeld B, Sonmez RGT, Kolber MJ, Contreras B, Harris R, Ozen S. Effect of hand position on EMG activity of the posterior shoulder musculature during a horizontal abduction exercise. J strength Cond Res. 2013;27(10):2644-2649. doi:10.1519/JSC.0b013e318281e1e9

15. Lehman GJ, Buchan DD, Lundy A, Myers N, Nalborczyk A. Variations in muscle activation levels during traditional latissimus dorsi weight training exercises: An experimental study. Dyn Med. 2004;3:4. doi:10.1186/1476-5918-3-4

16. Alenabi T, Jackson M, Tetreault P, Begon M. Electromyographic activity in the immobilized shoulder musculature during ipsilateral elbow, wrist, and finger movements while wearing a shoulder orthosis. J shoulder Elb Surg. 2013;22(10):1400-1407. doi:10.1016/j.jse.2013.04.007

17. Kuhtz-Buschbeck JP, Jing B. Activity of upper limb muscles during human walking. J Electromyogr Kinesiol. 2012;22(2):199206. doi:https://doi.org/10.1016/j.jelekin.2011.08.014

18. McNair PJ, Depledge J, Brettkelly M, Stanley SN. Verbal encouragement: effects on maximum effort voluntary muscle: action. Br J Sports Med. 1996;30(3):243-245.

19. Soares EG, Brown LE, Gomes WA, Corrêa DA, Serpa ÉP, da Silva JJ, et al. Comparison Between Pre-Exhaustion and Traditional Exercise Order on Muscle Activation and Performance in Trained Men. J Sports Sci Med. 2016;15(1):111-117.

20. Hermens HJ, Freriks B, Disselhorst-Klug C, Rau G. Development of recommendations for SEMG sensors and sensor placement procedures. J Electromyogr Kinesiol. 2000;10(5):361-374.

21. Escamilla RF, Babb E, DeWitt R, Jew P, Kelleher P, Burnham T, et al. Electromyographic analysis of traditional and nontraditional abdominal exercises: implications for rehabilitation and training. Phys Ther. 2006;86(5):656-671.

22. Konrad P. The abc of emg. A Pract Introd to Kinesiol Electromyogr. 2005;1:30-35.

23. Kristiansen M, Samani A, Vuillerme N, Madeleine P, Hansen EA. External and Internal Focus of Attention Increases Muscular Activation During Bench Press in Resistance-Trained Participants. J Strength Cond Res. 2018;32(9):2442-2451.
24. Hollmann, W.; Hettinger TH. Medicina Do Esporte. Ed. Manole.; 1983.

25. Nazário-de-Rezende F, Haddad EG, Sousa G da C, Agostini GG de, Nunes JED, Marocolo Jr. M. Déficit bilateral em exercício multiarticular para membros superiores. Rev Bras Med do Esporte. 2012;18:385-389.

26. Masuda K, Masuda T, Sadoyama T, Inaki M, Katsuta S. Changes in surface EMG parameters during static and dynamic fatiguing contractions. J Electromyogr Kinesiol. 1999;9(1):39-46.

27. Henneman E. Functional organization of motoneuron pools: the size-principle. Integr Nerv Syst. 1979:13-25.

28. Moritani T, Nagata A, Muro M. Electromyographic manifestations of muscular fatigue. Med Sci Sports Exerc. 1982;14(3):198-202.

\section{Acknowledgments}

The authors have no conflicts of interest to disclose. The authors also thank the Coordenação de Aperfeiçoamento de Pessoal de Nível Superior - Brazil (CAPES) for the financial support to run this project (Financing Code - 001).

\section{Corresponding author}

Matheus Machado Gomes

Escola de Educação Física e Esporte de Ribeirão Preto.

Universidade de São Paulo.

Av. Bandeirantes, 3900. CEP 14040-907.

Ribeirão Preto, SP, Brazil.

E-mail: mmgomes@usp.br

Manuscript received on May 6, 2019

Manuscript accepted on October 9, 2019

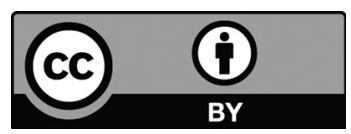

Motriz. The Journal of Physical Education. UNESP. Rio Claro, SP, Brazil eISSN: 1980-6574 - under a license Creative Commons - Version 4.0 